\title{
Short Review of Computational Models for Single Cell Deformation and Migration
}

\section{Vermolen FJ*}

Delft Institute of Applied Mathematics, Delft University of Technology, The Netherlands

\section{Short Communication}

This short review communication aims at enumerating several modeling efforts that have been performed to model cell migration and deformation. To optimize and improve medical treatments against diseases like cancer, ischemic wounds or pressure ulcers, it is of vital importance to understand the underlying biological mechanisms. Such biological mechanisms also take place on a cellular scale, where cells are known to migrate, proliferate, and differentiate and to die. Cell migration is necessary for constituent cells and immune cells to reach the damage area or carcinoma. The mechanisms for migration can have different natures, such as random walk, chemotaxis, haptotaxis, tensotaxis, durotaxis, or for instance thermotaxis. The latter five migration mechanisms are consequences of gradients of a chemical (such as a nutrient, hormone, or toxic agents) in a liquid, in a solid, local stresses, gradients of tissue stiffness or gradients of temperature.

Next to the vast experimental in vitro and even in vivo knowledge on migration and deformation of cells, computational models exist which turn biological insights into quantitative relations such that an experimental validation can be carried out. The developed computational models for cell deformation and migration have a varying nature. Roughly speaking, we distinguish the following spatial model classes:

\section{Cellular automata-models}

These fully discrete models are based on a distribution of the domain of computation into a lattice where all points contain a flag saying whether or not the lattice point is occupied by the cell. At each discrete time-step, a point has a certain probability to transform between being occupied or not occupied by a certain cell. This probability depends on environmental factors like chemical and/or mechanical environment. The cellular-Potts models form an important subset of this modeling class. One can also use the same lattice points as a grid on which one approximates field variables such as a concentration of a chemical [1]

\section{Semi-discrete models}

These models do not incorporate a mesh on the domain of computation to locate the cells, but merely contain a mesh on the surface (that is the membrane) of the cell. All points on the cell surface are connected via spring forces to their immediate neighbors and to the cell nucleus. The cells are allowed to deform and migrate via various processes such as random walk or any form of taxis (such as chemotaxis). On all the surface points, one computes the signal that influences the migration. One can use analytic relations, such as Green's Fundamental Solutions, or finite-element approximations for the field variables such as the concentration of a chemical [2]. An example of such a simulation is presented in Figure 1, where a cell deforms and migrates as a result of the concentration gradient of a chemical. This simulation can be used to model immune cells migrating towards pathogens that induce a field of biotic lactates. It is also noted that in [3] cell migration in relation to an electric stimulus is considered.

\section{Fully continuous models}

These models are based on the solution of partial differential equations that typically describe mechanical equilibrium. Such models can be based on Hooke's Law or on visco-elastic principles. Here the cell volume is distributed into mesh points, both internally and on the cell boundary. Some points on the cell boundary may sense a stimulus to migrate towards a certain direction as a result of an external field (for instance a chemical). The displacement will induce a strain-stress field in the whole cell, which affects the shape of the cell during its migration. These models typically involve heavy computations $[4,5]$ for instance.

Next to these modeling efforts, one can distinguish cellular migration models that actually contain trailing edges, or consider collective cell migration. Furthermore, cells are also known to pull or push on their environment, this process has also been modeled in several studies [6].

\section{References}

1. Van Oers RF, Rens EG, LaValley DJ, Reinhart-King CA, Merks RM (2014) Mechanical cell-matrix feedback explains pairwise and collective endothelial cell behavior in vitro. PLoS Comput Biol 10: e1003774.

2. Vermolen FJ, Gefen A (2013) A phenomenological model for chemicomechanically induced cell shape changes during migration and cell-cell contacts. Biomech Model Mechanobiol 12: 301-323.

3. Mousavi SJ, Doweidar MH, Doblaré M (2013) 3D computational modelling of cell migration: a mechano-chemo-thermo-electrotaxis approach. J Theor Biol 329: 64-73.

4. Borau C, Polacheck WJ, Kamm RD, Garcia-Aznar JM (2014) Probabilistic voxel-FE model for single cell motility in 3D. In Silico Cell and Tissue Science 1: 2

5. Madzvamuse A, George UZ (2013) The moving grid finite element method applied to cell movement and deformation. Finite Elements Analysis and Design 74: 76-92

6. Lemmon CA, Romer LH (2010) A predictive model of cell traction forces based on cell geometry. Biophys J 99: L78-80.
*Corresponding author: Vermolen FJ, Delft Institute of Applied Mathematics, Delft University of Technology, The Netherlands, Tel: +31 15278 8022; E-mail: F.J.Vermolen@tudelft.nl

Received May 15, 2015; Accepted June 17, 2015; Published June 19, 2015

Citation: Vermolen FJ (2015) Short Review of Computational Models for Single Cell Deformation and Migration. Single Cell Biol S1: 002. doi:10.4172/2168-9431.S1-002

Copyright: ( 2015 Vermolen FJ. This is an open-access article distributed under the terms of the Creative Commons Attribution License, which permits unrestricted use, distribution, and reproduction in any medium, provided the original author and source are credited. 
Citation: Vermolen FJ (2015) Short Review of Computational Models for Single Cell Deformation and Migration. Single Cell Biol S1: 002. doi:10.4172/21689431.S1-002

8

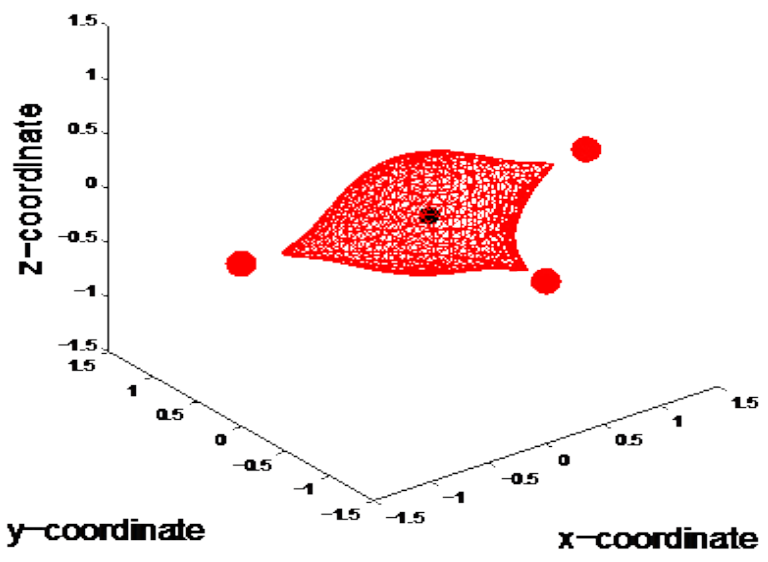

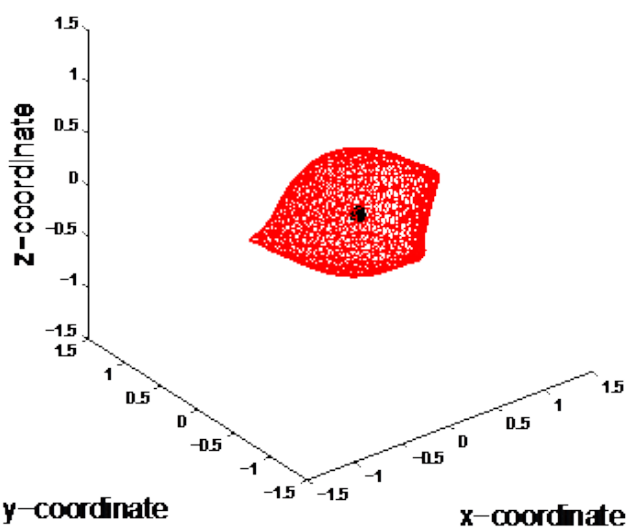

Figure 1: Snapshots of a simulation where a cell (red area) engulfs three pathogen sources (red heavy dots). The model was originally presented in Vermolen and Gefen [2]. The cell boundary is divided into mesh points. 